\title{
Activating KRAS mutations are characteristic of oncocytic sinonasal papilloma and associated sinonasal squamous cell carcinoma
}

\author{
Aaron M Udager,' Jonathan B McHugh,' Bryan L Betz,' Kathleen T Montone, ${ }^{2}$ Virginia A Livolsi, ${ }^{2}$ Raja R \\ Seethala, ${ }^{3}$ Evgeny Yakirevich, ${ }^{4} \mathrm{O}$ Hans Iwenofu, ${ }^{5}$ Bayardo Perez-Ordonez, ${ }^{6}$ Kathleen E DuRoss, ' Helmut C \\ Weigelin,' Megan S Lim, ${ }^{2}$ Kojo SJ Elenitoba-Johnson* and Noah A Brown'** \\ Department of Pathology, University of Michigan Health System, Ann Arbor, MI, USA \\ 2 Department of Pathology, Perelman School of Medicine at University of Pennsylvania, Philadelphia, PA, USA \\ 3 Department of Pathology, University of Pittsburgh Medical Center, Pittsburgh, PA, USA \\ 4 Department of Pathology, Brown University, Providence, RI, USA \\ 5 Department of Pathology, Ohio State University, Columbus, OH, USA \\ 6 Laboratory Medicine and Pathobiology, University of Toronto, Toronto, Ontario, Canada \\ *Correspondence to: NA Brown, MD, Department of Pathology, University of Michigan Medical School, I 107 Traverwood II, Suite C, 2910 Huron \\ Parkway, Ann Arbor, MI 48105, USA. E-mail: noahbr@umich.edu \\ Or KSJ Elenitoba-Johnson, MD, Department of Pathology, University of Pennsylvania Medical School, 609A Stellar Chance Laboratories, 420 Curie \\ Boulevard, Philadelphia, PA 1904, USA. E-mail: Kojo.Elenitoba-Johnson@uphs.upenn.edu
}

\begin{abstract}
Oncocytic sinonasal papillomas (OSPs) are benign tumours of the sinonasal tract, a subset of which are associated with synchronous or metachronous sinonasal squamous cell carcinoma (SNSCC). Activating EGFR mutations were recently identified in nearly 90\% of inverted sinonasal papillomas (ISPs) - a related tumour with distinct morphology. EGFR mutations were, however, not found in OSP, suggesting that different molecular alterations drive the oncogenesis of these tumours. In this study, tissue from 51 cases of OSP and five cases of OSP-associated SNSCC was obtained retrospectively from six institutions. Tissue was also obtained from 50 cases of ISP, 22 cases of ISP-associated SNSCC, ten cases of exophytic sinonasal papilloma (ESP), and 19 cases of SNSCC with no known papilloma association. Using targeted next-generation and conventional Sanger sequencing, we identified KRAS mutations in 51/51 (100\%) OSPs and 5/5 (100\%) OSP-associated SNSCCs. The somatic nature of KRAS mutations was confirmed in a subset of cases with matched germline DNA, and four matched pairs of OSP and concurrent associated SNSCC had concordant KRAS genotypes. In contrast, KRAS mutations were present in only one (5\%) SNSCC with no known papilloma association and none of the ISPs, ISP-associated SNSCCs, or ESPs. This is the first report of somatic KRAS mutations in OSP and OSP-associated SNSCC. The presence of identical mutations in OSP and concurrent associated SNSCC supports the putative role of OSP as a precursor to SNSCC, and the high frequency and specificity of KRAS mutations suggest that OSP and OSP-associated SNSCC are biologically distinct from other similar sinonasal tumours. The identification of KRAS mutations in all studied OSP cases represents an important development in our understanding of the pathogenesis of this disease and may have implications for diagnosis and therapy.

Copyright $@ 2016$ Pathological Society of Great Britain and Ireland. Published by John Wiley \& Sons, Ltd.
\end{abstract}

Keywords: KRAS; oncocytic; papilloma; squamous cell carcinoma; sinonasal; Schneiderian

Received II April 2016; Revised 19 May 2016; Accepted 23 May 2016

No conflicts of interest were declared

\section{Introduction}

Sinonasal (Schneiderian) papillomas, which account for approximately $1-5 \%$ of sinonasal tumours, include inverted sinonasal papilloma (ISP; approximately 62\%), exophytic sinonasal papilloma (ESP; approximately $32 \%$ ), and oncocytic sinonasal papilloma (OSP; approximately 6\%) [1]. Similar to ISP, OSP usually arises from the lateral nasal wall or paranasal sinuses and is associated with synchronous or metachronous sinonasal squamous cell carcinoma (SNSCC) in a subset of cases (up to 15\%). OSP, however, has a distinctive histomorphology composed of an epithelial proliferation of bland columnar cells with eosinophilic cytoplasm, intraepithelial mucin cysts/neutrophilic abscesses, and mixed exophytic and endophytic growth patterns [1,2]. Importantly, the genetic basis for OSP and associated SNSCC is unknown. Recently, highly-prevalent activating EGFR mutations were identified in the vast majority of ISPs [3]. These mutations were also commonly present in ISP-associated SNSCC but not in ESP, OSP, or SNSCC without a known papilloma association. These 

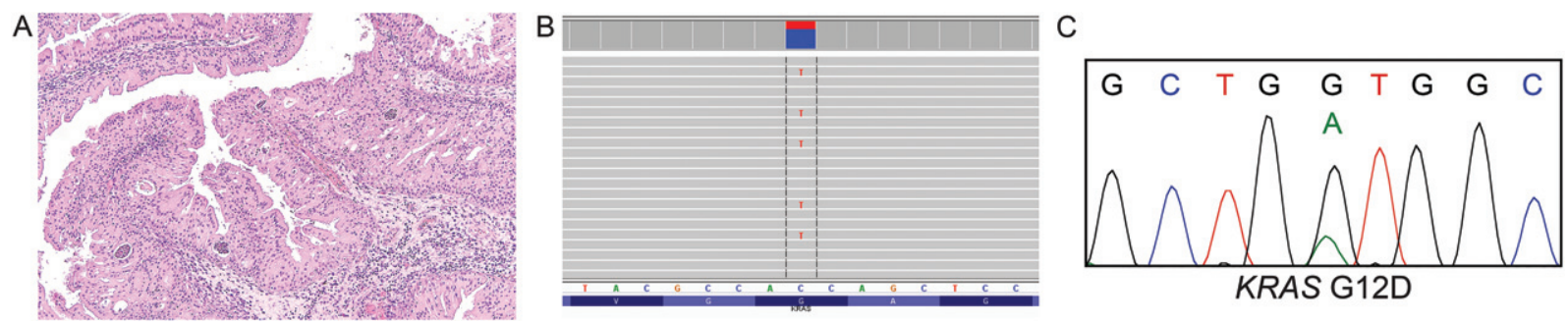

Figure 1. KRAS G12D mutation in OSP detected by targeted next-generation sequencing. (A) OSP initially evaluated by targeted next-generation sequencing. (B) Integrated Genomics Viewer representation of the KRAS G12D mutation detected by next-generation sequencing (shown in reverse complement). (C) Sequence electropherogram showing confirmation of KRAS G12D mutation by Sanger sequencing.

data suggest that the ISP/ISP-associated SNSCC disease spectrum is biologically distinct from other sinonasal lesions, including OSP. Therefore, in this study, we sought to identify possible recurrent oncogenic drivers in OSP and explore the genetic relationship of OSP to associated SNSCC.

\section{Materials and methods}

\section{Specimens}

With institutional review board approval, formalinfixed, paraffin-embedded (FFPE) tissue was obtained from six institutions including 51 OSPs and 5 OSP-associated SNSCCs from 52 unique patients. In addition, FFPE tissue from 50 ISPs, 22 ISP-associated SNSCCs, 10 ESPs, and 19 SNSCCs without a known papilloma association was obtained from the University of Michigan, as described previously [3]. All diagnoses were confirmed centrally by an experienced head and neck pathologist (JBM). DNA was extracted using the Pinpoint Slide DNA Isolation System (Zymo Research, Irvine, CA, USA), according to the manufacturer's instructions. For a subset of OSP patients, constitutional
DNA was also extracted from adjacent normal sinonasal tissue.

\section{DNA sequencing}

Targeted next-generation sequencing was performed as described previously [3]. In brief, sequencing libraries were generated from $10 \mathrm{ng}$ of extracted DNA using the Ion AmpliSeq Cancer Hotspot Panel v2 (Thermo Fisher Scientific, Waltham, MA, USA); variants were called using Torrent Suite 4.0.2 and assessed using the Broad Institute's Integrated Genomics Viewer (IGV 2.3). Conventional bidirectional Sanger sequencing of $K R A S$ exons 2 and 3 and EGFR exons 18, 19, 20, and 21 was performed using nested sequencing primers, as described previously [3].

\section{Results and discussion}

In order to identify possible pathogenic mutations, two cases of OSP were evaluated using targeted next-generation sequencing. In both cases, a guanineto-adenine transition in codon 12 , resulting in a

\section{Oncocytic Sinonasal Papillomas KRAS Mutations}



\section{OSP-Associated Sinonasal SCC KRAS Mutations}

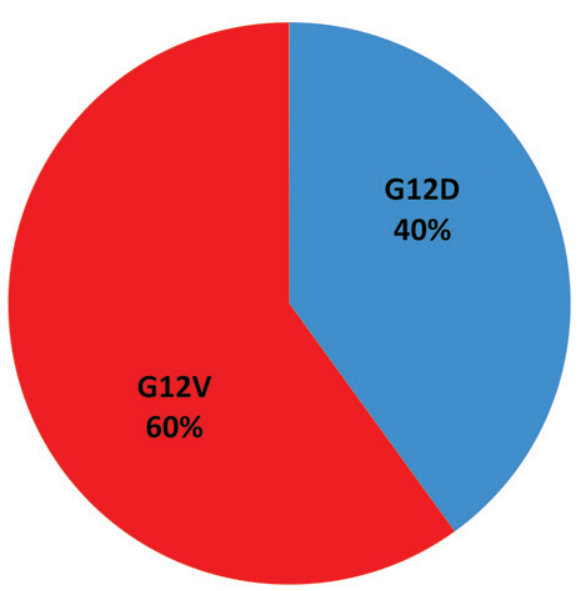

Figure 2. Frequencies of KRAS mutations in OSP and OSP-associated SNSCC. 
A

\section{Oncocytic Sinonasal Papilloma}
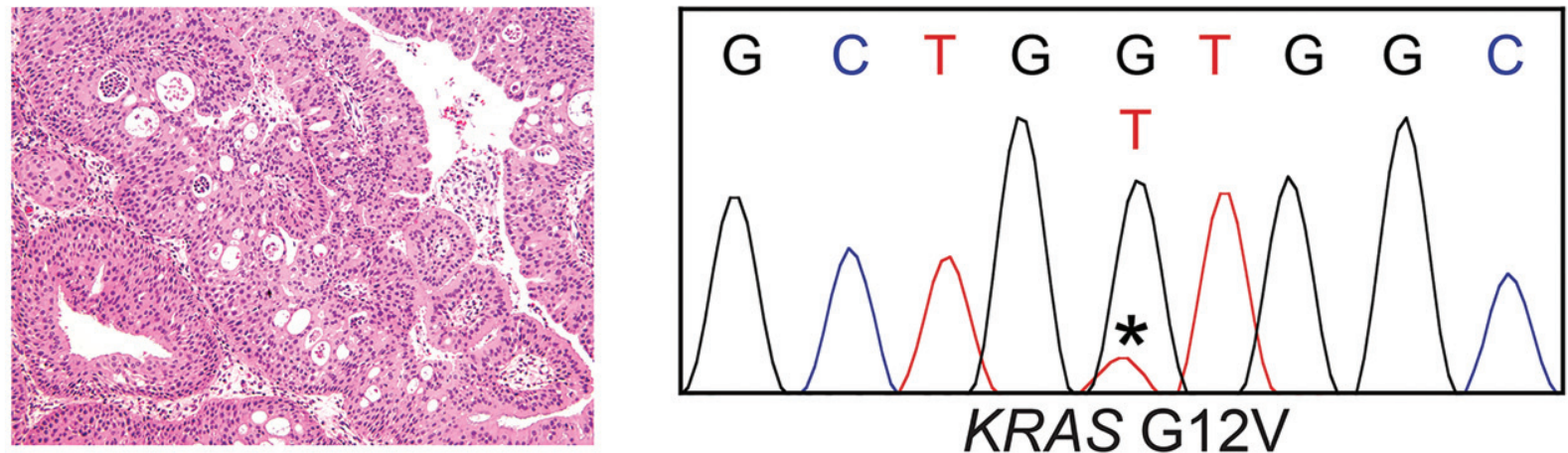

Matched OSP-Associated Sinonasal Squamous Cell Carcinoma
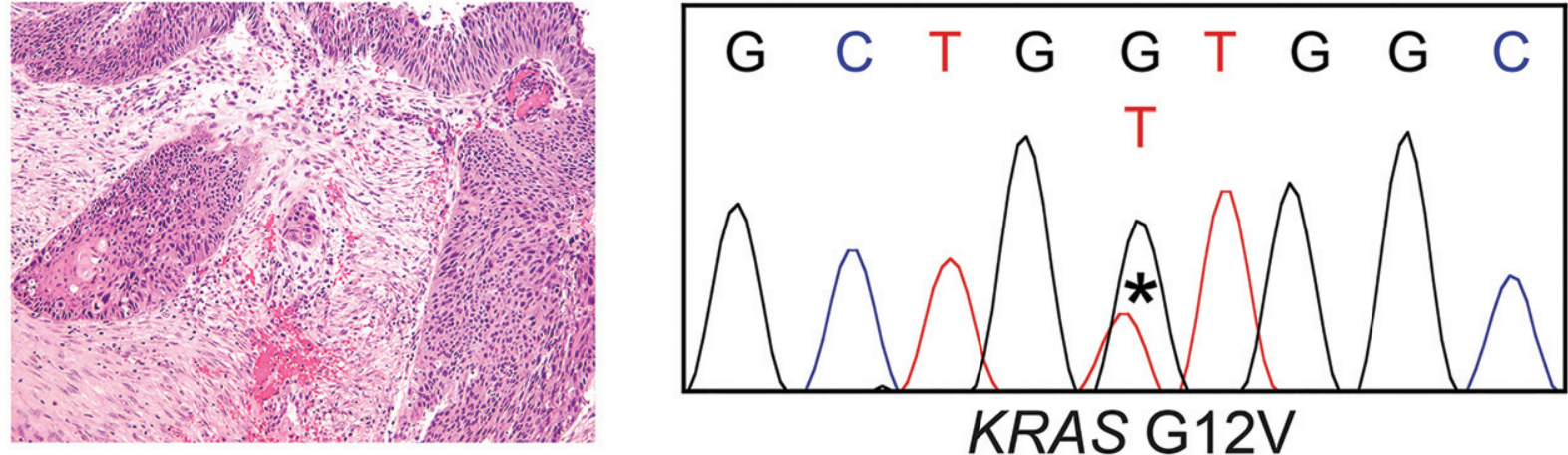

B

\begin{tabular}{ccccc} 
& \multicolumn{2}{c}{ OSP KRAS Genotype } & & \multicolumn{2}{c}{ OSP-associated SNSCC KRAS Genotype } \\
\cline { 5 - 6 } Patient & cDNA & Protein & CDNA & Protein \\
\hline 1 & $35 \mathrm{G}>\mathrm{A}$ & $\mathrm{G} 12 \mathrm{D}$ & $35 \mathrm{G}>\mathrm{A}$ & $\mathrm{G} 12 \mathrm{D}$ \\
2 & $35 \mathrm{G}>\mathrm{T}$ & $\mathrm{G} 12 \mathrm{~V}$ & $35 \mathrm{G}>\mathrm{T}$ & $\mathrm{G} 12 \mathrm{~V}$ \\
3 & $35 \mathrm{G}>\mathrm{T}$ & $\mathrm{G} 12 \mathrm{~V}$ & $35 \mathrm{G}>\mathrm{T}$ & $\mathrm{G} 12 \mathrm{~V}$ \\
4 & $35 \mathrm{G}>\mathrm{A}$ & $\mathrm{G} 12 \mathrm{D}$ & $35 \mathrm{G}>\mathrm{A}$ & $\mathrm{G} 12 \mathrm{D}$ \\
\hline
\end{tabular}

Figure 3. Molecular relationship between OSP and associated SNSCC. (A) Histopathology and KRAS exon 2 sequence electropherograms for a separately extracted OSP (top) and concurrent associated SNSCC (bottom) from one patient demonstrating the same mutation (G12V). (B) KRAS genotypes for concurrent OSP and associated SNSCC from four unique patients.

glycine-to-aspartic acid missense substitution (G12D), was identified in the KRAS gene (Figure 1). No other mutations in any of the other 49 genes on the Ion AmpliSeq Cancer Hotspot Panel v2 were identified, and in both cases, the KRAS G12D mutation was confirmed by Sanger sequencing and demonstrated to be somatic by evaluating constitutional DNA extracted from adjacent normal sinonasal tissue.

To determine the incidence of KRAS mutations in OSP and OSP-associated SNSCC, we evaluated a large, multi-institutional cohort of tumours by Sanger sequencing of exons 2 and 3 (see supplementary material, Table S1). Overall, $100 \%$ of OSPs $(n=51)$ harboured KRAS mutations, either in codon 12 or in codon 61 (Figure 2). Importantly, all identified mutations have previously been described in a number of common malignancies including colon, pancreas, and lung cancer [4-6]. In addition, all mutations have been shown to be oncogenic through constitutive activation of the KRAS protein and the mitogen-activated protein kinase (MAPK) pathway [7-12]. The universal observation of $K R A S$ mutations in OSP suggests that these mutations represent an essential oncogenic event for these tumours.

Similar to OSP, $100 \%$ of SNSCCs (including one adenosquamous carcinoma) arising in patients with concurrent or metachronous OSP $(n=5)$ harboured $K R A S$ mutations (Figure 2). While OSP and ISP are occasionally observed in patients who present with SNSCC, the molecular relationship between these papillomas and associated SNSCC is uncertain. In a previous study [3], we compared EGFR genotypes in matched pairs of ISP and associated SNSCC. In spite of the heterogeneity of EGFR mutations observed among tumours from different patients, identical EGFR genotypes were observed in matched ISP and associated SNSCC pairs. In the current study, material was 


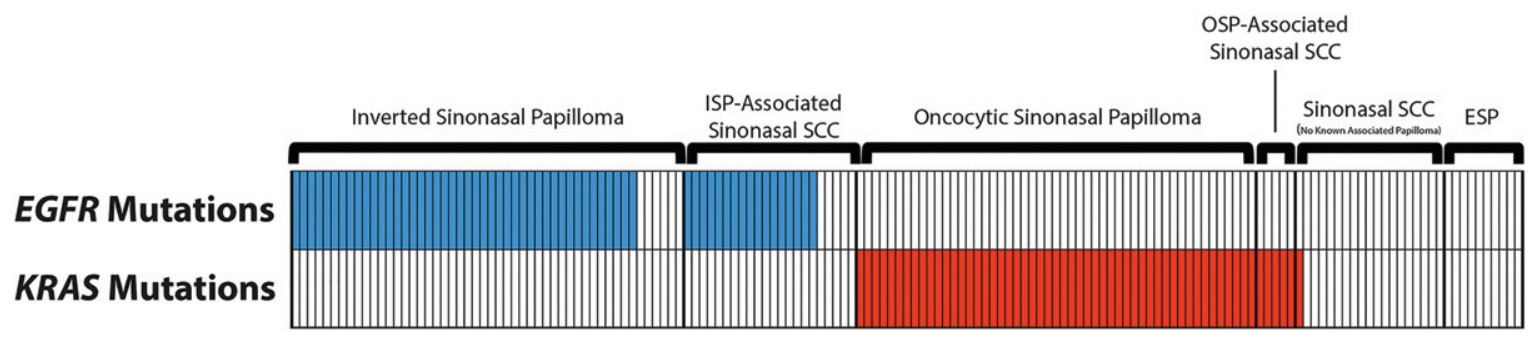

Figure 4. KRAS and EGFR genotypes of sinonasal papillomas and squamous cell carcinoma. KRAS mutations are a universal finding in OSP and OSP-associated SNSCC but are not identified in ISP, ISP-associated SNSCC or ESP and are uncommon in SNSCC without a known papilloma association. In contrast, EGFR mutations are only detected in ISP and ISP-associated SNSCC [3].

available from four patients with OSP and concurrent associated SNSCC. Similar to our previous findings in ISP and associated SNSCC, separately extracted DNA from each matched OSP and associated SNSCC pair demonstrated identical KRAS genotypes (Figure 3). While the number of matched cases of OSP and associated SNSCC in this study is small, these results provide evidence to support the premise that sinonasal papillomas - including OSP - are precursor lesions for associated SNSCC. Indeed, although the mechanisms of malignant progression from sinonasal papilloma to SNSCC are not well understood, high-risk human papillomavirus (HPV) infection is thought to play a role in a subset of ISP-associated SNSCCs [13].

While KRAS mutations have been reported in a significant proportion of intestinal-type sinonasal adenocarcinomas [14-17], KRAS mutations are very uncommon in squamous cell carcinomas of the head and neck [18-20] and have only rarely been reported in SNSCC $[14,21]$. To determine the frequency of KRAS mutations in other similar sinonasal lesions, we evaluated a large cohort using Sanger sequencing (Figure 4 and supplementary material, Table S1). No KRAS mutations were identified in other sinonasal papillomas, including ISP $(n=50)$ and ESP $(n=5)$. In addition, KRAS mutations were not identified in ISP-associated SNSCC $(n=22)$ and were found in only one case (5\%) of SNSCC without a known papilloma association $(n=19)$. Between two previous studies, only one KRAS mutation (G12A) was detected among more than 100 unselected SNSCCs $[14,21]$. It is uncertain if KRAS mutations truly do occur at a low frequency in de novo SNSCC or if the apparent absence of an associated OSP in these cases reflects insufficient clinical information, incomplete sampling, or overgrowth of an OSP by the associated SNSCC. Regardless, the universal presence of KRAS mutations in oncocytic sinonasal tumours (OSP and OSP-associated SNSCC), the absence of these mutations in other sinonasal papillomas, and the rarity of these mutations in other similar sinonasal lesions, however, suggest that KRAS mutations could be perceived as a disease-defining molecular feature of sinonasal oncocytic tumours.

The identification of KRAS mutations in OSP and OSP-associated SNSCC may also have important implications for therapy. In colorectal cancer, KRAS mutations are an important negative predictor of response to targeted anti-EGFR therapy [22]. Likewise,
KRAS mutations in non-small cell lung cancer are mutually exclusive with $E G F R$ mutations and portend a poor response to EGFR inhibitor therapy [23]. The absence of any benefit from EGFR-targeted therapy in tumours with KRAS mutations is thought to reflect the fact that KRAS is downstream of EGFR in the growth factor signalling pathway. Although not currently in wide use, a number of small molecule MEK inhibitors are in various phases of clinical trials and may someday be useful for the treatment of tumours with RAS mutations [24].

Collectively, the results of this study, our previously published data, and other previous studies provide a working model for oncogenic drivers in sinonasal papillomas: (1) OSP harbours KRAS mutations; (2) ISP contains EGFR mutations (and/or low-risk HPV infection); and (3) ESP is associated with low-risk HPV infection $[1,3,25]$. The precise reason for this genotype-phenotype correlation is not readily apparent, particularly in light of the fact that activating mutations in KRAS and EGFR are likely to have similar functional consequences related to MAPK pathway activation. The phenotypic differences between ISP and OSP may reflect a difference in the cell of origin, associated secondary mutations, and/or activation of distinct gene expression programmes. Alternatively (or in addition), oncocytic tumours are characterized by an aberrant number of mitochondria imparting a swollen appearance with abundant eosinophilic cytoplasm [26]. In many oncocytic tumours, the increase in mitochondria is linked to mitochondrial dysfunction through a variety of mechanisms [27]. Because KRAS mutations have been linked to mitochondrial dysfunction, these mutations may contribute to the oncocytic appearance of OSP [28]. Indeed, KRAS mutations in follicular thyroid lesions are associated with oncocytic change, in contrast to those with mutations in NRAS or HRAS - related genes in the growth factor signalling pathway [29].

Overall, our studies implicate a previously unknown and prominent role for activating KRAS mutations in the pathogenesis of oncocytic sinonasal tumours and suggest that KRAS mutations may be a disease-defining molecular feature of sinonasal oncocytic tumours. The presence of KRAS mutations in these tumours indicates that EGFR-targeted therapy is likely to be ineffective, while other treatments currently in clinical trials may one day play a therapeutic role. 


\section{Acknowledgment}

This work was supported by the University of Michigan Health System (Department of Pathology, Divisions of Anatomic Pathology and Clinical Pathology).

\section{Author contribution statement}

The authors contributed in the following way: AMU, MSL, KSJEJ, NAB: study design; AMU, JBM, KM, VAL, RRS, EY, OHI, BPO, KED, HCW: data collection; AMU, JBM, NAB: data analysis and data interpretation; AMU, NAB: literature search; AMU, BLB, NAB: generation of figures; AMU, BLB, MSL, KSJEJ: writing of the manuscript.

\section{References}

1. Barnes L. Schneiderian papillomas and nonsalivary glandular neoplasms of the head and neck. Mod Pathol 2002; 15: 279-297.

2. Barnes L, Bedetti C. Oncocytic Schneiderian papilloma: a reappraisal of cylindrical cell papilloma of the sinonasal tract. Hum Pathol 1984; 15: $344-351$.

3. Udager AM, Rolland DC, McHugh JB, et al. High-frequency targetable EGFR mutations in sinonasal squamous cell carcinomas arising from inverted sinonasal papilloma. Cancer Res 2015; 75: 2600-2606.

4. Cancer Genome Atlas Network. Comprehensive molecular characterization of human colon and rectal cancer. Nature 2012; 487: 330-337.

5. Cancer Genome Atlas Research Network. Comprehensive molecular profiling of lung adenocarcinoma. Nature 2014; 511: 543-550.

6. Biankin AV, Waddell N, Kassahn KS, et al. Pancreatic cancer genomes reveal aberrations in axon guidance pathway genes. Nature 2012; 491: 399-405.

7. Davies BR, Logie A, McKay JS, et al. AZD6244 (ARRY-142886), a potent inhibitor of mitogen-activated protein kinase/extracellular signal-regulated kinase kinase 1/2 kinases: mechanism of action in vivo, pharmacokinetic/pharmacodynamic relationship, and potential for combination in preclinical models. Mol Cancer Ther 2007; 6: 2209-2219.

8. Haigis KM, Kendall KR, Wang Y, et al. Differential effects of oncogenic K-Ras and N-Ras on proliferation, differentiation and tumor progression in the colon. Nature Genet 2008; 40: 600-608.

9. Fatrai S, van Gosliga D, Han L, et al. KRAS(G12V) enhances proliferation and initiates myelomonocytic differentiation in human stem/progenitor cells via intrinsic and extrinsic pathways. $J$ Biol Chem 2011; 286: 6061-6070.

10. Modest DP, Camaj P, Heinemann V, et al. KRAS allele-specific activity of sunitinib in an isogenic disease model of colorectal cancer. J Cancer Res Clin Oncol 2013; 139: 953-961.
11. Park JT, Johnson N, Liu S, et al. Differential in vivo tumorigenicity of diverse KRAS mutations in vertebrate pancreas: a comprehensive survey. Oncogene 2015; 34: 2801-2806.

12. Stolze B, Reinhart S, Bullinger L, et al. Comparative analysis of KRAS codon 12, 13, 18, 61, and 117 mutations using human MCF10A isogenic cell lines. Sci Rep 2015; 5: 8535.

13. Strojan P, Ferlito A, Lund VJ, et al. Sinonasal inverted papilloma associated with malignancy: the role of human papillomavirus infection and its implications for radiotherapy. Oral Oncol 2012; 48: $216-218$.

14. Lopez F, Garcia Inclan C, Perez-Escuredo J, et al. KRAS and BRAF mutations in sinonasal cancer. Oral Oncol 2012; 48: 692-697.

15. Garcia-Inclan C, Lopez F, Perez-Escuredo J, et al. EGFR status and $K R A S / B R A F$ mutations in intestinal-type sinonasal adenocarcinomas. Cell Oncol 2012; 35: 443-450.

16. Projetti F, Durand K, Chaunavel A, et al. Epidermal growth factor receptor expression and KRAS and BRAF mutations: study of 39 sinonasal intestinal-type adenocarcinomas. Hum Pathol 2013; 44: 2116-2125.

17. Szablewski V, Solassol J, Poizat F, et al. EGFR expression and KRAS and BRAF mutational status in intestinal-type sinonasal adenocarcinoma. Int J Mol Sci 2013; 14: 5170-5181.

18. Agrawal N, Frederick MJ, Pickering CR, et al. Exome sequencing of head and neck squamous cell carcinoma reveals inactivating mutations in NOTCH1. Science 2011; 333: 1154-1157.

19. Stransky N, Egloff AM, Tward AD, et al. The mutational landscape of head and neck squamous cell carcinoma. Science 2011; 333: $1157-1160$.

20. Cancer Genome Atlas Network. Comprehensive genomic characterization of head and neck squamous cell carcinomas. Nature 2015; 517: $576-582$.

21. Bornholdt J, Hansen J, Steiniche T, et al. K-ras mutations in sinonasal cancers in relation to wood dust exposure. BMC Cancer 2008; 8: 53 .

22. Arrington AK, Heinrich EL, Lee W, et al. Prognostic and predictive roles of KRAS mutation in colorectal cancer. Int J Mol Sci 2012; 13: 12153-12168.

23. Timar J. The clinical relevance of KRAS gene mutation in non-small-cell lung cancer. Curr Opin Oncol 2014; 26: 138-144.

24. Neuzillet C, Tijeras-Raballand A, de Mestier L, et al. MEK in cancer and cancer therapy. Pharmacol Ther 2014; 141: 160-171.

25. Udager AM, McHugh JB, Elenitoba-Johnson KS, et al. EGFR mutations in sinonasal squamous tumors: oncogenic and therapeutic implications. Oncoscience 2015; 2: 908-909.

26. Chang A, Harawi SJ. Oncocytes, oncocytosis, and oncocytic tumors. Pathol Annu 1992; 27 (Pt 1): 263-304.

27. Gasparre G, Romeo G, Rugolo M, et al. Learning from oncocytic tumors: why choose inefficient mitochondria? Biochim Biophys Acta 2011; 1807: 633-642.

28. $\mathrm{Hu} \mathrm{Y}, \mathrm{Lu} \mathrm{W}$, Chen $\mathrm{G}$, et al. K-ras ${ }^{\mathrm{G} 12 \mathrm{~V}}$ transformation leads to mitochondrial dysfunction and a metabolic switch from oxidative phosphorylation to glycolysis. Cell Res 2012; 22: 399-412.

29. Radkay LA, Chiosea SI, Seethala RR, et al. Thyroid nodules with KRAS mutations are different from nodules with NRAS and HRAS mutations with regard to cytopathologic and histopathologic outcome characteristics. Cancer Cytopathol 2014; 122: 873-882.

\section{SUPPLEMENTARY MATERIAL ON THE INTERNET}

The following supporting information may be found in the online version of this article:

Table S1. KRAS and EGFR sequencing results in a large cohort of sinonasal papillomas and squamous cell carcinomas 